\title{
The freeze-shut of a convectively cooled parallel plate channel subjected to laminar internal liquid flow*
}

\author{
B. Weigand and G. Ruß, Darmstadt, FRG
}

\begin{abstract}
The paper presents an approximative solution for the time dependent development of the ice layers at the cooled walls inside a parallel plate channel. The upper and the lower wall of the channel are cooled by an uniform external convection. By assuming a constant pressure drop across the channel, the freeze-shut of the planar channel could be calculated approximately. It was found out that the origin of the freezing fronts moves upstream during the ice layer growth. Furthermore a simple criterion is presented to predict whether a given system will lead to blockade.
\end{abstract}

\section{Das Zufrieren eines konvektiv gekühlten, ebenen Kanals} bei laminarer Durchströmung

Zusammenfassung. Die vorliegende Arbeit stellt eine Approximationslösung vor, die das instationäre Wachstum der Eisschichten in einem ebenen, laminar durchströmten Kanal beschreibt. Die obere und die untere Wand des Kanals werden hierbei konvektiv gekühlt. Unter der Annahme eines zeitlich konstanten Druckverlustes im Kanal ist es möglich, das instationäre Verhalten der Erstarrungsfronten, bis hin zur Blockade des Kanals, approximativ zu berechnen. Als ein Ergebnis der Arbeit ergibt sich, daß der örtliche Beginn der erstarrten Schicht an der Kanalwand mit dicker werdenden Eisschichten stromaufwärts wandert. Weiterhin wird ein Kriterium angegeben, das es erlaubt, a priori darüber zu entscheiden, ob das System bei den vorliegenden Verhältnissen zufriert.

\section{Nomenclature}

a thermal diffusivity

$B$ dimensionless freezing parameter $=k_{s} /\left(k_{L} \theta_{\infty}\right)$

$D(\tau) \quad$ volume ratio $=V(\tau=0) / V(\tau)$

$F_{n} \quad$ eigenfunctions, Eq. (11)

$F_{O} \quad$ Fourier number $=\mathrm{ta}_{\mathrm{s}} / \mathrm{h}^{2}$

$G_{m} \quad$ eigenfunctions, Eq. (21)

$h$ distance from centerline to the wall

$k$ thermal conductivity

$L \quad$ channel length

$L_{s} \quad$ length of the solidification-free zone

$p \quad$ pressure

Pr Prandtl number

$r_{s} \quad$ heat of fusion

$R e_{h}(\tau) \quad$ Reynolds number based on $h=V(\tau) /\left(2 v_{L}\right)$

$R e_{4 h}(\tau)$ Reynolds number based on the hydraulic diameter $(4 h)$

Ste Stefan number $=c_{s}\left(T_{F}-T_{\infty}\right) / r_{s}$

$T \quad$ temperature

$T_{F} \quad$ freezing temperature of the liquid

* Dedicated to Prof. Dr.-Ing. H. Beer's 60th birthday

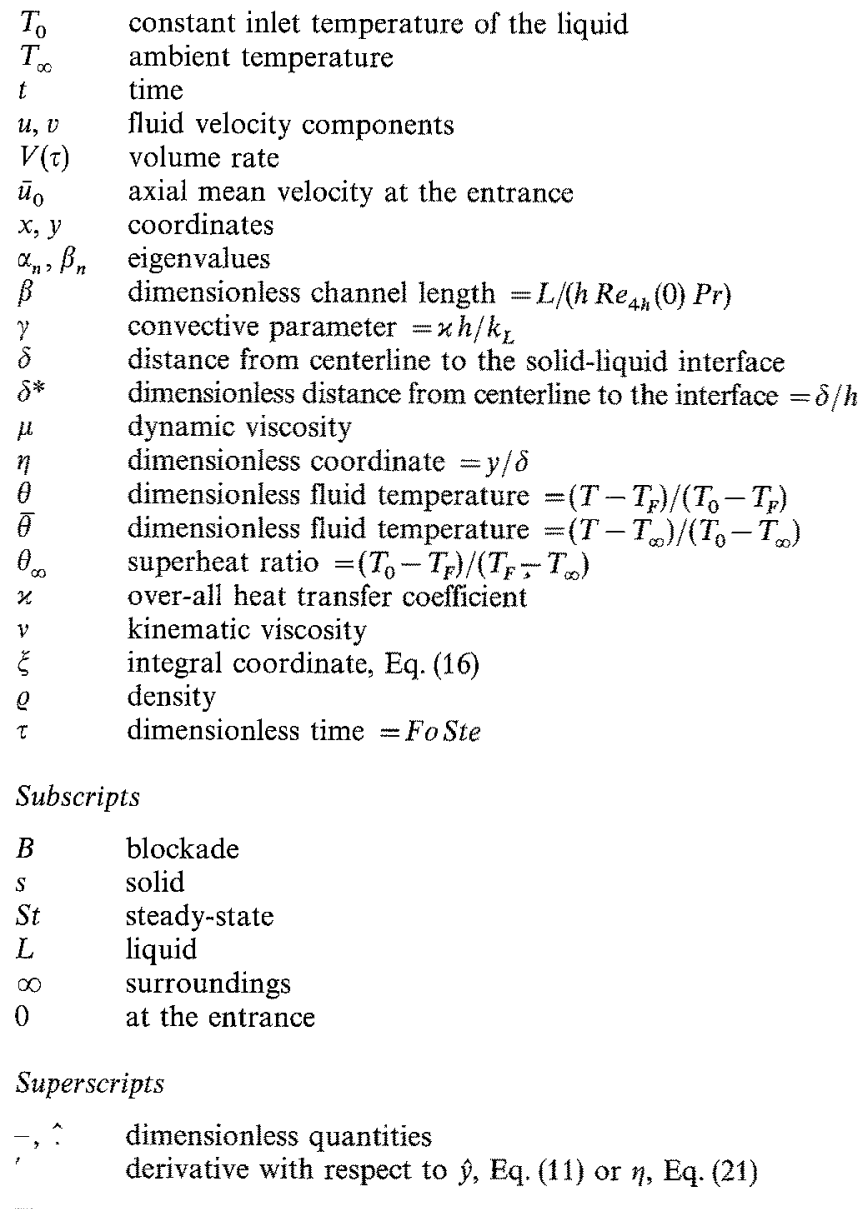

\section{Introduction}

The freezing of liquids in forced laminar flow inside circular tubes or parallel plate channels is of technical importance. This type of solidification process arises for example in the flow of liquid metals in parallel plate heat exchangers. Furthermore, it can be observed in many casting operations, where molten material is poured through channels and noz- 
zles, the wall of which are initially below the freezing temperature of the flowing material.

Many theoretical and experimental studies have been performed for fluid flow in circular tubes and channels with internal solidification [1-5]. The seminal papers in this field are due to Zerkle and Sunderland [1] and Lee and Zerkle [2]. In the papers $[1-5]$ the mass flow rate at the inlet of the cooled test section is assumed to be constant. Therefore, these studies preclude by definition freezing shut of the apparatus.

However, in some situations the pressure drop across a system, rather than the mass flow rate, may remain constant as freezing occurs in a portion of the system. This could arise for a flow between large reservoirs, between a reservoir and the atmosphere, or possibly in a system where liquid is circulated by a centrifugal pump. A system of this type may freeze shut. If the pressure drop across a system remains constant, the mass flow rate must decrease with increasing thickness of the ice layers. Hence, the tube or the channel may either freeze shut or the mass flow rate may be reduced to a steadystate value for which no further solidification occurs.

Sampson and Gibson [6] derived a mathematical model for predicting the time dependent development of the frozen crust inside a cooled tube with laminar liquid flow and a constant wall temperature. They assumed a constant pressure drop across the cooled pipe section. Furthermore, they supposed the axial velocity profile to be parabolic throughout the whole chill region. Their analysis lead to a criterion which can be used to predict conditions under which blockade will occur for the case of uniform wall temperature. This criterion was checked with some experimental results due to Des Ruisseaux and Zerkle [6] and a generally good agreement was observed.

For the case of a parallel plate channel no investigations are known in literature, which deal with the blockade of the system. Furthermore, no investigation was undertaken in the past which incorporates the influence of external cooling of the pipe or the channel on the freeze-shut of the system. Therefore, the object of the current investigation is to analyze the freezing of laminar liquid flow in a parallel plate channel subjected to external convection. By assuming that the pressure drop across the test section can be considered as constant, which is in analogy to the work of Sampson and Gibson [6], the time dependent development of the ice layers and the freeze shut of the system will be calculated approximately.

\section{Analysis}

Fig. 1 shows the geometric configuration and the coordinate system for a planar symmetric duct. The laminar fluid flow enters the cooled test section at $x=0$ with a fully developed velocity profile and with the constant temperature $T_{0}$. At the origin of the time coordinate, $t=0$, the temperature of the ambient is suddenly lowered for $x \geq 0$ from $T_{0}$ to the temper-

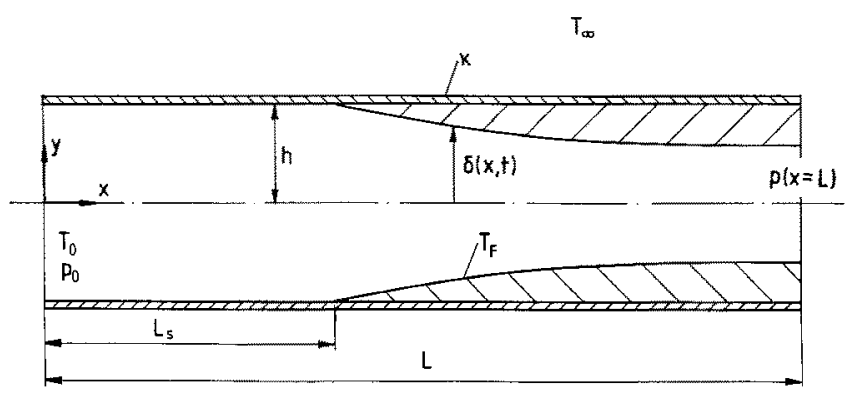

Fig. 1. Physical model and coordinate system

ature $T_{\infty}$, which is lower than the freezing temperature $T_{F}$ of the fluid. Heat transfer takes place by conduction through the channel walls and by convection to the surroundings. Therefore, the liquid is cooled as it flows through the channel and eventually, at some downstream location $\left(x=L_{s}\right)$, the freezing temperature $T_{F}$ of the fluid is reached at the walls and solidification begins. Thus, the thermal entrance region consists of a solidification-free zone and a freezing zone where the solid layer increases in thickness monotonously along the channel walls.

It is assumed that the physical properties are constant and axial heat conduction, viscous energy dissipation and free convection are negligible. Further, quasi steady-state conditions will be assumed. This is justified because the typical time scale of the fluid motion is much shorter than the typical time scale of the freezing process. Finally, it will be assumed that the pressure difference $p(x=L)-p_{0}$ is constant all the time. This supposition is made in order to model the blockade of the planar channel in analogy to the work of Sampson and Gibson [6]. It might not be right for some technical equipments, but it will be shown that $p(x=L)-p_{0}$ can be considered as an arbitrary function of the volume rate in the channel without any complications.

\subsection{Velocity distributions}

In the solidification-free zone $0 \leq x \leq L_{s}$ the established Plane Poiseuille flow at $x=0$ will be conserved,

$u=\frac{3}{4} \frac{V(t)}{h}\left(1-\left(\frac{y}{h}\right)^{2}\right)$,

where $V(t)$ denotes the volume rate per unit of width. By deriving Eq. (1), quasi steady-state conditions are assumed.

In the freezing zone $L_{s} \leq x \leq L$ the velocity distribution in the liquid region can be approximated by

$$
\begin{aligned}
& u=\frac{3 V(t)}{4 \delta(x, t)}\left(1-\left(\frac{y}{\delta(x, t)}\right)^{2}\right), \\
& v=\frac{y}{\delta(x, t)} u \frac{\partial \delta}{\partial x} .
\end{aligned}
$$

The velocity profiles according to Eqs. (2) and (3) were obtained from the continuity and momentum equations by 
assuming that $R e_{h} \partial \delta / \partial x$ tends to zero, as it was shown by Weigand and Beer [5]. However, it was recognized in [5] that the supposition of the velocity profile, given by Eqs. (2) and (3), leads to a good approximation for the ice layer thickness at the channel walls for moderate values of the cooling parameter $(B<10)$.

\subsection{Pressure drop and volume rate in the channel}

Inserting the velocity distributions according to Eqs. (1)-(3) into the momentum equations and neglecting terms of the order $\partial \delta / \partial x$ results in the following equation for the pressure drop across the channel

$p(L)-p_{0}=-\int_{L_{s}}^{L} \frac{3}{2} \mu_{L} \frac{V(t)}{\delta^{3}(x, t)} d x-\frac{3}{2} \mu_{L} L_{s} \frac{V(t)}{h^{3}}$.

If $p(L)-p_{0}$ is constant, Eq. (4) can be solved explicitly for $V(t)$. The volume rate is found to be

$V(t)=\frac{2\left(p_{0}-p(L)\right)}{3 \mu_{L}\left(\int_{L_{s}}^{L} \frac{d x}{\delta^{3}(x, t)}+\frac{L_{s}}{h^{3}}\right)}$.

If the pressure drop $\left(p_{0}-p(L)\right)$ is an arbitrary function of $V(t)$, Eq. (4) is an implicit equation which combines the shape of the ice with the reduction in flow rate during the growth of the frozen crust.

\subsection{The energy equation}

The energy equation of the liquid region can be simplified by invoking the upper mentioned assumptions. One obtaines

$u \frac{\partial T}{\partial x}+v \frac{\partial T}{\partial y}=a_{L} \frac{\partial^{2} T}{\partial y^{2}}$.

Eq. (6) can be solved separately in the solidification-free zone $\left(x \leq L_{s}\right)$ and in the freezing zone $\left(x \geq L_{s}\right)$.

\subsubsection{The solidification-free zone $\left(0 \leq x \leq L_{s}\right)$}

The energy equation for the parabolic flow of liquid in the thermal entrance region of the parallel plate channel is given in dimensionless form as

$$
\frac{3}{2}\left(1-\hat{y}^{2}\right) \frac{\partial \bar{\theta}}{\partial \bar{x}}=D(\tau) \frac{\partial^{2} \bar{\theta}}{\partial \hat{y}^{2}}
$$

with the boundary conditions

$$
\begin{array}{ll}
\bar{x}=0: & \bar{\theta}=1, \\
\hat{y}=0: & \frac{\partial \bar{\theta}}{\partial \hat{y}}=0, \\
\hat{y}=1: & \frac{\partial \bar{\theta}}{\partial \hat{y}}=-\gamma \bar{\theta} .
\end{array}
$$

In Eqs. (7) and (8) the following dimensionless quantities are used

$$
\begin{aligned}
& R e_{h}(0)=\frac{V(\tau=0)}{2 v_{L}}, \quad D(\tau)=\frac{\mathrm{V}(\tau=0)}{V(\tau)}=\frac{R e_{h}(\tau=0)}{R e_{h}(\tau)}, \\
& \bar{x}=\frac{x}{h} \frac{1}{R e_{h}(0) P r}, \quad \hat{y}=\frac{y}{h}, \quad \tau=\text { FoSte }, \quad \operatorname{Pr}=\frac{v_{L}}{a_{L}}, \\
& \bar{\theta}=\frac{T-T_{\infty}}{T_{0}-T_{\infty}}, \quad \gamma=\frac{x h}{k_{L}}, \quad F o=\frac{t a_{s}}{h^{2}}, \quad S t e=\frac{c_{s}\left(T_{F}-T_{\infty}\right)}{r_{s}} .
\end{aligned}
$$

The parameter $\gamma$, which appears in Eq. (8) represents the effect of external cooling. As it can be seen from Eq. (8) $\gamma=0$ denotes the case of an adiabatic channel, while $\gamma \rightarrow \infty$ represents the case of an uniform wall temperature $T_{\infty}$ at the channel walls. The function $D(\tau)$ is a volume rate ratio. With increasing ice layer thickness, the volume rate $V(\tau)$ is reduced, as it can be seen from Eq. (5). Therefore, $D(\tau)$ increases with growing values of $\tau$.

It is obvious that the time $\tau$ appears only as a parameter in Eq. (7). Hence, the energy equation (7) can be solved for a fixed time $\tau_{1}$ by the method of separation of variables [8]. After some routine algebra one obtaines

$\bar{\theta}=\sum_{n=1}^{\infty} A_{n} F_{n}(\hat{y}) \exp \left(-\frac{\alpha_{n}^{2}}{4} D(\tau) \bar{x}\right)$.

The constants $A_{n}$ as well as the eigenvalues $\alpha_{n}$ can be found in literature [9] for various values of $\gamma$. The eigenfunctions $F_{n}(\hat{y})$, appearing in Eq. (10), satisfy the Sturm-Liouville problem

$F_{n}^{\prime \prime}(\hat{y})+\frac{3}{8} \alpha_{n}^{2}\left(1-\hat{y}^{2}\right) F_{n}(\hat{y})=0$

in conjunction with the boundary conditions

$F_{n}^{\prime}(0)=0, \quad F_{n}^{\prime}(1)+\gamma F_{n}(1)=0$

and an arbitrary normalizing condition

$F_{n}(0)=1$.

In Eqs. (11) and (12) ' denotes the derivation of the function $F_{n}$ with respect to $\hat{y}$.

To determine the location $x=L_{s}$ at which the surface temperature becomes equal to the freezing temperature of the liquid, it is only necessary to set $\hat{y}=1$ and $T=T_{F}$ in Eq. (10). The following implicit equation is obtained for the determination of $\bar{x}_{s}$

$\frac{T_{F}-T_{\infty}}{T_{0}-T_{\infty}}=\frac{1}{1+\theta_{\infty}}=\sum_{n=1}^{\infty} A_{n} F_{n}(1) \exp \left(-\frac{\alpha_{n}^{2}}{4} D(\tau) \bar{x}_{s}\right)$.

The left hand side of Eq. (14) is a constant. Therefore, the sum on the right hand side of the equation must be constant, too. This could only be satisfied, if

$D(\tau) \bar{x}_{\mathrm{s}}=$ const.

for given values of $\gamma$ and $\theta_{\infty}$. Eq. (15) shows an interesting consequence of the assumption of a constant pressure drop across the test section. If the ice layer increases in thickness, 


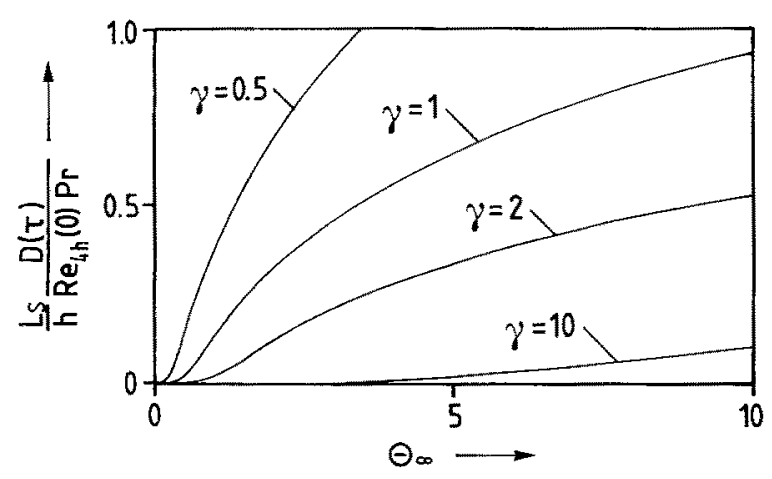

Fig. 2. Influence of the external convection parameter $\gamma$ and the superheat ratio $\theta_{\infty}$ on the origin of the ice layers

the function $D(\tau)$ increases because of the decreasing flow rate (see Eq. (9)). Hence, the axial distance $\bar{x}_{s}$ decreases with growing ice layer thickness, which means that the axial point at which freezing starts at the channel walls moves upstream. From a physical point of view the movement of the origin of the frozen crust can easily be understood. If the layers grow, the flow rate at the entrance of the test section decreases, which is obvious from Eq. (5). Therefore, the fluid loses more heat by flowing through the cooled parallel plate channel and the freezing temperature of the fluid at the channel walls is reached for smaller values of the axial coordinate. Fig. 2 shows the influence of external convection on the axial position of the origin of the ice layers. For a constant value of the parameter $\theta_{\infty}$, which is a superheat ratio, the origin of the freezing fronts is shifted to lower values of the axial coordinate for growing values of $\gamma$. This is obvious, because $\gamma \rightarrow \infty$ represents the case of constant wall temperature, for which the origin of the freezing fronts coincides with $x=0$.

\subsubsection{The freezing zone $\left(L_{s} \leq x \leq L\right)$}

Inserting the approximation for the velocity profile in the liquid region, given by Eqs. (2) and (3), into the energy equation (6) and applying the coordinate transformation

$\eta=\frac{y}{\delta}, \quad \xi=\int_{\bar{x}_{s}}^{\bar{x}} \frac{d \alpha}{\delta^{*}(\tau, \alpha)}$

to the energy equation, the following partial differential equation can be obtained

$\frac{3}{2}\left(1-\eta^{2}\right) \frac{\partial \theta}{\partial \xi}=D(\tau) \frac{\partial^{2} \theta}{\partial \eta^{2}}$

where the dimensionless quantities

$\theta=\frac{T-T_{F}}{T_{0}-T_{F}}, \quad \delta^{*}=\frac{\delta}{h}$

are used. The origin of the coordinate $\xi$, defined by Eq. (16), corresponds with the value $\bar{x}_{s}$ of the axial coordinate $\bar{x}$.
Eq. (17) has to be solved in accordance with the following boundary conditions

$$
\begin{aligned}
& \xi=0: \quad \theta=\frac{1+\theta_{\infty}}{\theta_{\infty}} \bar{\theta}\left(\bar{x}_{s}\right)-\frac{1}{\theta_{\infty}}, \\
& \eta=0: \quad \frac{\partial \theta}{\partial \eta}=0, \\
& \eta=1: \quad \theta=0 .
\end{aligned}
$$

The boundary condition for $\xi=0$ states the fact that the temperature distribution at this axial position is prescribed by Eq. (10).

The solution of Eq. (17) in conjunction with the boundary conditions Eq. (19) can be derived easily using the method of separation of variables. The temperature distribution $\theta$ in the liquid region is found to be

$\theta=\sum_{m=1}^{\infty} B_{m} G_{m}(\eta) \exp \left(-\frac{\beta_{m}^{2}}{4} D(\tau) \xi\right)$.

The eigenfunctions $G_{m}(\eta)$ are the solutions of the SturmLiouville problem

$G_{m}^{\prime \prime}(\eta)+\frac{3}{8}\left(1-\eta^{2}\right) \beta_{m}^{2} G_{m}(\eta)=0$

with the boundary conditions

$G_{m}^{\prime}(0)=0, \quad G_{m}(1)=0$

and an arbitrary normalizing condition

$G_{m}(1)=1$.

The eigenvalues $\beta_{m}$ can be found in literature [10]. The constants $B_{m}$, which appear in Eq. (20), can be calculated analytically by using the orthogonality relations of the eigenfunctions $G_{m}(\eta)$ and $F_{n}(\hat{y})$. After some algebra, one finally obtains

$B_{m}=\frac{\left(\frac{1+\theta_{\infty}}{\theta_{\infty}}\right) \sum_{n=1}^{\infty} A_{n} \frac{G_{m}^{\prime}(1) F_{n}(1)}{\alpha_{n}^{2}-\beta_{m}^{2}} \exp \left(-\frac{\alpha_{n}^{2}}{4} D(\tau) \bar{x}_{s}\right)+\frac{1}{\theta_{\infty}} \frac{G_{m}^{\prime}(1)}{\beta_{m}^{2}}}{\frac{3}{8} \int_{0}^{1}\left(1-\eta^{2}\right) G_{m}^{2}(\eta) d \eta}$

The eigenfunctions $F_{n}(\hat{y})$ and $G_{m}(\eta)$, appearing in Eq. (11) and Eq. (21) were calculated numerically with the help of a fourth order Runge-Kutta method.

\subsubsection{Temperature distribution in the solid region}

Assuming constant properties in the solid region and negligible axial conduction, the heat conduction equation for the solid-phase reduces, for quasi steady-state conditions, to

$\frac{\partial^{2} T_{s}}{\partial y^{2}}=0$ 
The associate boundary conditions are

$y=\delta(x, t): \quad T_{s}=T_{F}$,

$y=h: \quad k_{s} \frac{\partial T_{s}}{\partial y}+x\left(T_{s}-T_{\infty}\right)=0$.

Integrating Eq. (25) according to the boundary conditions, Eq. (26), results in the following expression for the temperature distribution in the spolid region

$T_{s}=T_{F}+\frac{\left(T_{F}-T_{\infty}\right)(y-\delta)}{\delta-h-k_{s} / \varkappa}$.

\subsection{The growth of the solid crust}

The time dependent development of the ice layers at the channel walls will be calculated from the interface energy equation which adopts the following form if terms proportional to $(\partial \delta / \partial x)^{2}$ are neglected

$\left.k_{s} \frac{\partial T_{s}}{\partial y}\right|_{y=\delta}-\left.k_{L} \frac{\partial T}{\partial y}\right|_{y=\delta}=\varrho_{s} r_{s} \frac{\partial \delta}{\partial t}$.

Eq. (28) states the fact that the heat conducted in the solid plus the heat arising from phase change equals the heat transported from the liquid to the interface. Introducing the dimensionless quantities given by Eq. (9) and Eq. (18) and using Eq. (20), Eq. (28) results in

$$
\begin{aligned}
\delta^{*}\left(\frac{\partial \delta^{*}}{\partial \tau}\right)_{\bar{x}}= & \frac{\delta^{*}}{\delta^{*}-1-\frac{k_{s}}{k_{L}} \frac{1}{\gamma}} \\
& -\frac{1}{B} \sum_{m=1}^{\infty} B_{m} G_{m}^{\prime}(1) \exp \left(-\frac{\beta_{m}^{2}}{4} D(\tau) \xi\right) .
\end{aligned}
$$

The function $D(\tau)$ is given in dimensionless form by

$D(\tau)=\frac{1}{4 \beta}\left[\int_{\bar{x} s}^{4 \beta} \frac{d \bar{x}}{\delta^{* 3}}-\bar{x}_{s}\right]$

where $\beta$ denotes the dimensionless channel length, defined as

$\beta=\frac{L}{h} \frac{1}{R e_{4 h}(0) P r}$.

The quantity $B$ in Eq. (29) denotes the dimensionless freezing parameter

$B=\frac{k_{s}}{k_{L}} \frac{1}{\theta_{\infty}}=\frac{k_{s}}{k_{L}} \frac{T_{F}-T_{\infty}}{T_{0}-T_{F}}$.

Eq. (32) elucidates that $B$ is proportional to $1 / \theta_{\infty}$, where $\theta_{\infty}$ denotes the superheat ratio of the fluid.

Eq. (29) is a strongly nonlinear integro-differential equation for $\delta^{*}$ which has to be solved in conjunction with the initial condition on $\delta^{*}$ that

$\delta^{*}(\tau=0)=1$.
The calculation of the frozen layer for different times can be performed in the following manner: Initially the constants $B_{m} G_{m}^{\prime}(1)$ and $\beta_{m}^{2}$, appearing in the interface energy equation (29), have to be calculated. This can be done by solving the eigenvalue problems according to Eqs. (11)-(13) and Eqs. (21)-(23). The solution of this equations was obtained numerically with the help of a fourth order Runge-Kutta process. As mentioned before, the eigenvalues $\alpha_{n}$ and $\beta_{n}$ can also be found in literature $[9,10]$. If the eigenvalues and the eigenfunctions are known, the constant $D(\tau) \vec{x}_{s}$ according to Eq. (15), can be calculated numerically from Eq. (14). For $\tau=0$ the function $D(0)=1$ and the origin of the ice layers is determined by Eq. (15).

The numerical solution of the integro-differential equation (29) was performed with the aid of a Runge-Kutta method for systems of differential equations. After integrating Eq. (29) at fixed values of $\bar{x}$, the local free channel height $\delta^{*}$ for $\tau+\Delta \tau$ is known. Therefore, the volume rate ratio $D(\tau+\Delta \tau)$, can be calculated from Eq. (30). With the known value of $D(\tau+\Delta \tau)$, the new origin of the ice layer is fixed by Eq. (15). The time step $\Delta \tau$ for the calculations was chosen after some numerical experiments as approximately 0.001 . For the evaluation of the sum in Eq. (29) taking nearly 50 terms guaranteed sufficient accuracy for the case of a convectively cooled channel, while for the case of constant wall temperature $(\gamma \rightarrow \infty)$ approximately 200 terms have to be used.

The calculations were stoped, if the channel blocked or if steady-state conditions were reached. The blockade of the channel was defined by

$\delta^{*}\left(\bar{x}=\frac{L}{h} \frac{1}{R e_{h}(0) P r}, \tau\right)<10^{-2}$

whereas steady-state conditions were reached if

$\left|\frac{\partial \delta^{*}}{\partial \bar{\tau}}\right|_{\bar{x}}<10^{-4}$ for $\quad \bar{x}=\frac{L}{h} \frac{1}{R e_{h}(0) P r}$

was satisfied.

\section{Results and discussion}

\section{3.t Constant wall temperature}

The case of an uniform temperature at the channel walls is obtained for $\gamma \rightarrow \infty$ from Eq. (8). If the temperature at the channel walls is maintained at the constant value $T_{\infty}$, which is lower than the freezing temperature of the liquid, the origin of the freezing fronts at the walls is fixed at $x=0$ for all times and the length of the solidification-free zone is identically zero.

Fig. 3 shows the time dependent development of the frozen crust at the channel walls for $B=3.5$ and a dimensionless channel length of $\beta=8 \cdot 10^{-4}$. The figure elucidates that the channel blocks after a dimensionless time $\tau \geq 1.41$. The following two figures show phase planes of the value of the 
crust at the end of the channel. It is obvious from the plots that the velocity of the ice-water interface at the end of the channel depends strongly on the value of $\delta^{*}$. For the case of an uniform wall temperature, $\left|\partial \delta^{*} / \partial \tau\right|$ tends to infinity if $\delta^{*} \rightarrow 1$. This can be seen from the interface energy equation (29) for $\gamma \rightarrow \infty$. For $B=3.5$ it can be observed in Fig. 4 that $\left|\partial \delta^{*} / \partial \tau\right|$ reaches a minimum for $\delta^{*}$ approximately 0.3 . In Fig. 3 it can be seen that the ice layer thickness at the end of the channel increases rapidly for low values of the dimensionless time, while the frozen crust grows slowly for $0.34 \leq \tau \leq 1.1$. For greater values of the dimensionless time, the velocity $\left|\partial \delta^{*} / \partial \tau\right|$ increases, as it can be seen in the phase plane, Fig. 4. For $\tau \geq 1.41$ the channel blocks and $\partial \delta^{*} / \partial \tau$ adopts at finite value at the end of the channel. In the case of the freeze-shut of a circular pipe $\left|\partial \delta^{*} / \partial \tau\right|$ tends to infinity for $\delta^{*} \rightarrow 0$ (see Sampson and Gibson [6]).

A other interesting detail can be seen in Fig. 5. The phase plane for $B=14.2$ shows that two steady-state solutions $\left(\partial \delta^{*} / \partial \tau=0\right)$ may exist. This phenomena can be attributed to the strongly nonlinear character of the interface energy equation (29), which is a nonlinear integro-differential equation. The double solution was also noted by Sampson and Gibson [6] in the case of internal freezing in pipe flow. With the help of a linear stability theory, it can be shown in analogy to [6], that only the thinner ice layer will be stable, whereas the thicker ice layer is found to be unstable against finite perturbations. From a physical point of view this result is easily understood, because the frozen crust will not encroach further into the liquid region if $\partial \delta^{*} / \partial \tau$ reaches the value zero. Therefore, the thicker ice layer never exists in reality.

\subsection{Convectively cooled channel}

For the calculation of the following ice layers the ratio of the thermal conductivities $k_{\mathrm{s}} / k_{L}$ was taken to be 4 in order to reduce the free parameters entering the problem. The value taken for $k_{s} / k_{L}$ corresponds, as a good approximation, to the internal flow of water inside the channel.

Fig. 6 shows the development of the frozen crust for $B=10$ and a convection parameter $\gamma=10$. It can be observed that a steady-state ice layer is reached for the chosen parameters. In Fig. 7, the phase plane of the value of the crust at the end of the channel is plotted for $\beta=10^{-4}$ and a convection parameter $\gamma=10$. The figure elucidates that $\left|\partial \delta^{*} / \partial \tau\right|$ decreases continuously for $B=10$. Further, it can be observed that $\partial \delta^{*} / \partial \tau$ adopts a finite value for $\delta^{*}=1$ for a convectively cooled channel. This fact can be understood by recognizing that the denominator of the first term on the right hand side of Eq. (29) reaches the value $-k_{s} / k_{L} 1 / \gamma$ for $\delta^{*}=1$. Furthermore, it can be observed that the velocity of the interface, $\left|\partial \delta^{*} / \partial \tau\right|$, at the end of the channel decreases with decreasing values of $\gamma$. This is due to the smaller amount of heat which can be convected to the ambient for smaller values of $\gamma$.

Fig. 8 and Fig. 9 show the development of freezing fronts for a lower convection parameter $\gamma=2$ and two different freezing parameters $B$. It can be seen that steady-state condi-

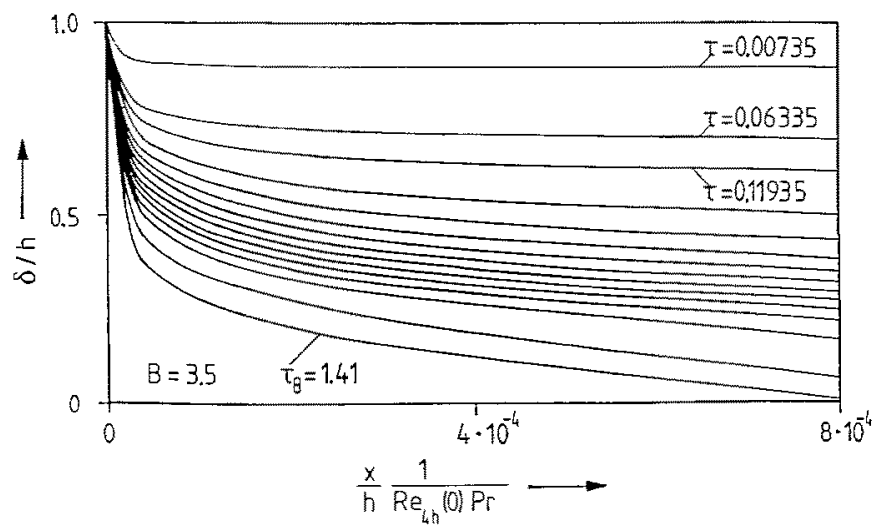

Fig. 3. Transient development of the frozen crust as a function of the axial coordinate for constant wall temperature (curves for $\tau>0.34335$ for equidistant time steps $\Delta \tau=0.112$ )

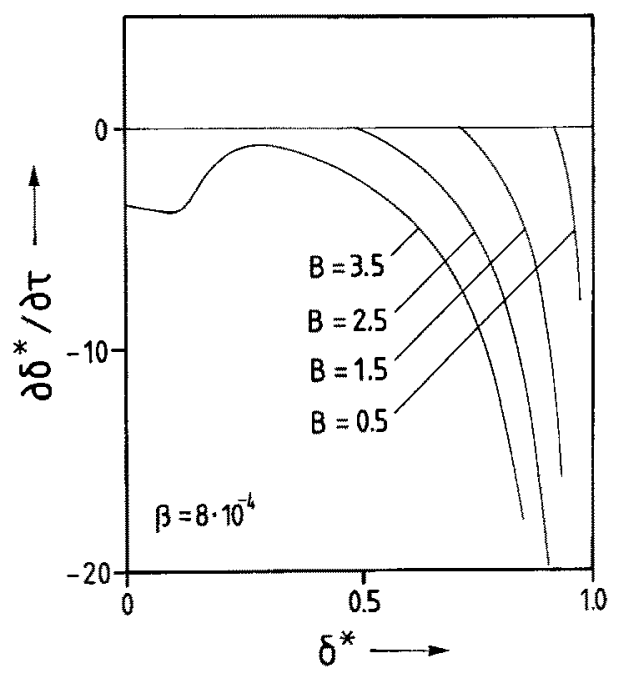

Fig. 4. Phase plane for $\delta^{*}$ at the end of the channel for constant wall temperature

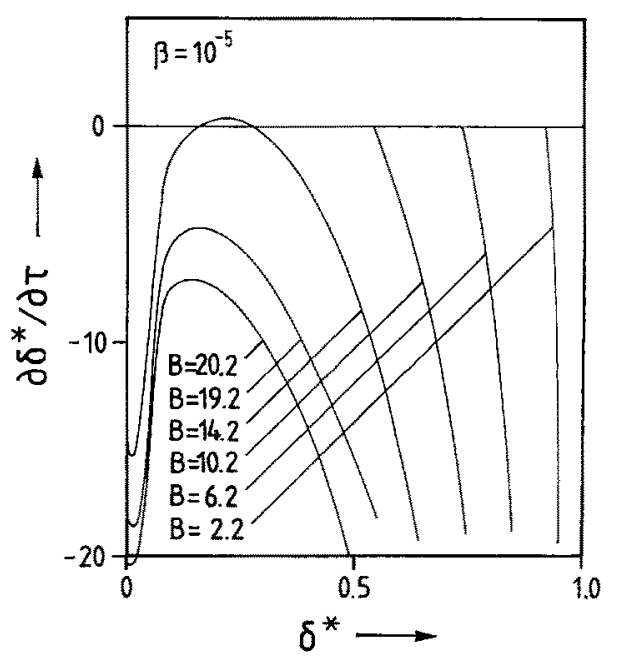

Fig. 5. Phase plane for $\delta^{*}$ at the end of the channel for constant wall temperature 


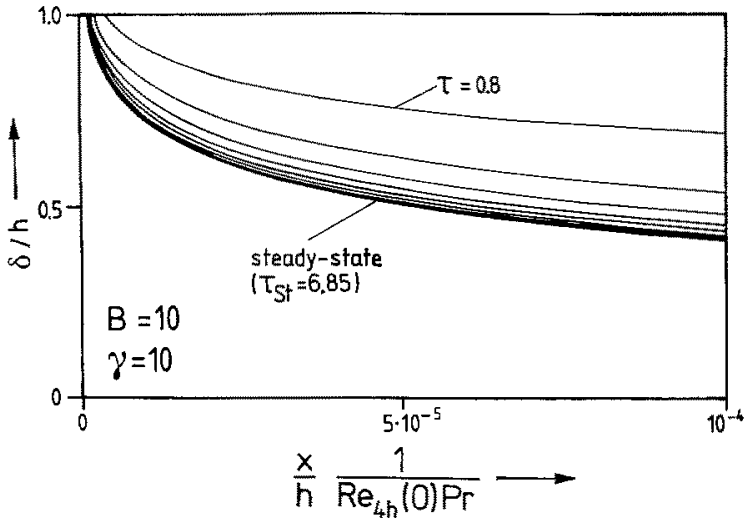

Fig. 6. Transient development of the frozen crust as a function of the axial coordinate (curves for equidistant time steps $\Delta \tau=0.8$ )

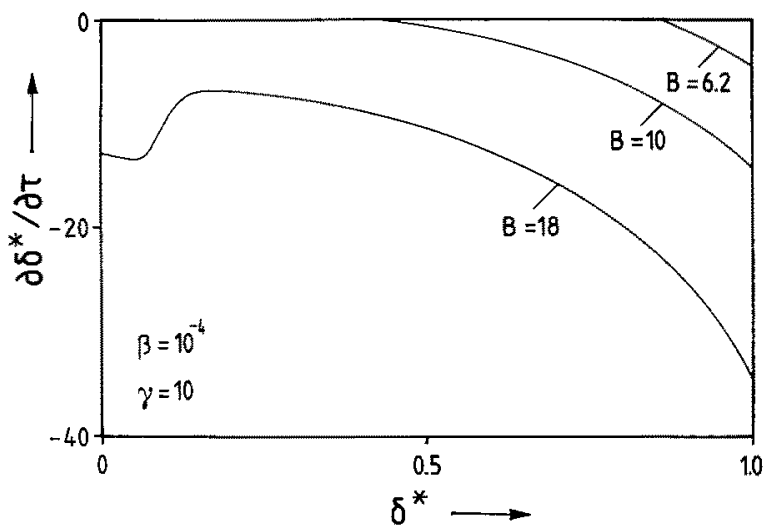

Fig. 7. Phase plane for $\delta^{*}$ at the end of the channel for a convectively cooled duct

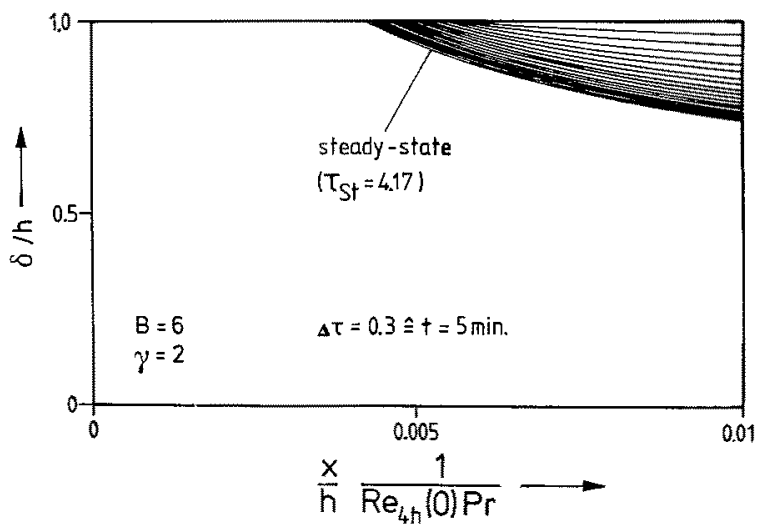

Fig. 8. Transient development of the frozen crust as a function of the axial coordinate (curves for equidistant time steps $\Delta \tau=0.3$ )

tions will be obtained for $B=6$, while the channel blocks for $B=10$. Fig. 8 and Fig. 9 visualize the movement of the origin of the frozen crust. To give the reader a more realistic insight into the real time scale of the movement of the origin, Fig. 8 indicates a relation between the dimensionless time $\tau$ and $t$

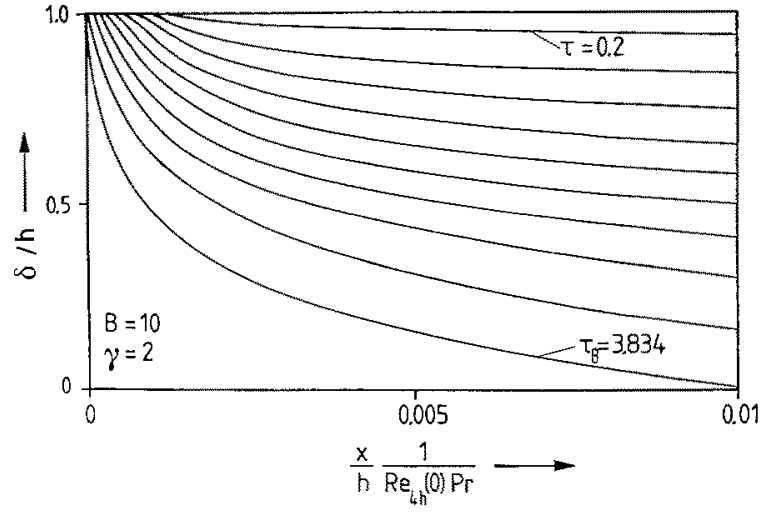

Fig. 9. Transient development of the frozen crust as a function of the axial coordinate (curves for equidistant time steps $\Delta t=0.2$ )

( $h=12 \mathrm{~mm}, T_{F}-T_{\infty}=14 \mathrm{~K}$ ). If we assume $R e_{4 h}(0)=1000$ and $\operatorname{Pr}=10$, the origin of the ice layers moves with a mean velocity of approximately $2 \mathrm{~mm} / \mathrm{min}$ in the upstream direction.

Fig. 10 shows a phase plane of the value of the crust at the end of the channel for the dimensionless channel length $\beta=0.01$ and $\gamma=2$. By comparing the Figs. 5, 7 and 10 it is obvious that the value of $\left|\partial \delta^{*} / \partial \tau\right|$ at the end of the channel decreases with decreasing values of $\gamma$. The freezing process is dominated by heat conduction through the frozen crust for low values of $\gamma$. This fact can be seen in Fig. 11, where the freeze-shut of a channel is shown for the parameters $B=10$, $\beta=1$ and $\gamma=0.5$. It is elucidated that the ice layers form nearly parallel lines to the $\bar{x}$ axis for $\bar{x}>0.5$. The reason for this behaviour can be declared by examining Eq. (29). Neglecting the second term on the right hand side of Eq. (29), which denotes the heat transfered by convection from the fluid to the solid crust, and integrating the resulting expression, results in

$\delta^{*}=a-\sqrt{2 \tau+(1-a)^{2}}$

where the quantity $a$ is defined by

$a=1+\frac{k_{s}}{k_{L}} \frac{1}{\gamma}$

Calculating $\delta^{*}$ at the end of the cooled channel from Eq. (36) with the parameters given in Fig. 11, one obtaines for $\tau=5$; $\delta_{\mathrm{Eq},(36)}^{*}=0.4$ and for $\tau=8.1: \delta_{\mathrm{Eq} .(36)}^{*}=0.045$, whereas the numerical predicted values, shown in Fig. 11 are $\tau=5$ : $\delta^{*}=0.4$ and for $\tau=8.1: \delta^{*}=0.048$. The comparison shows that the development of the frozen crust for low values of $\gamma$ is dominated by heat conduction, whereas convection in the liquid influences the shape of the freezing front only for small values of the axial coordinate.

Finally, Fig. 12 shows a "blockade diagram", which makes it possible to predict a priori if a system will lead to blockade or if steady-state conditions can be obtained. The curves for constant values of the convective parameter $\gamma$, drawn in Fig. 12, devide the regions for which the channel blocks 


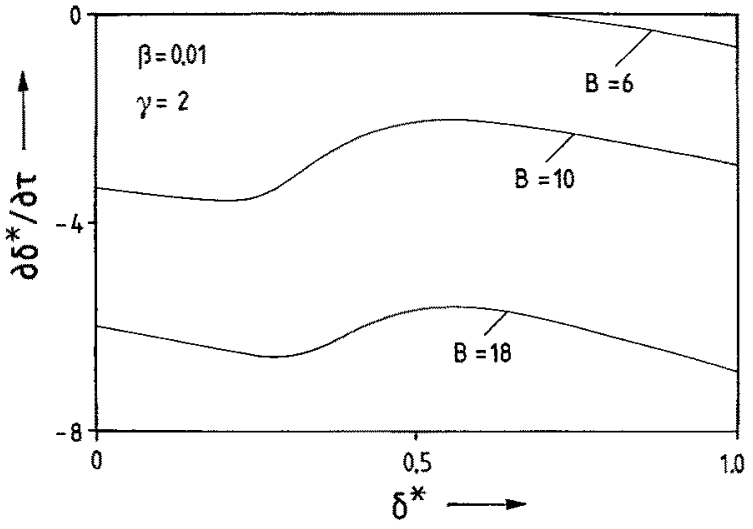

Fig. 10. Phase plane for $\delta^{*}$ at the end of the channel for a convectively cooled channel

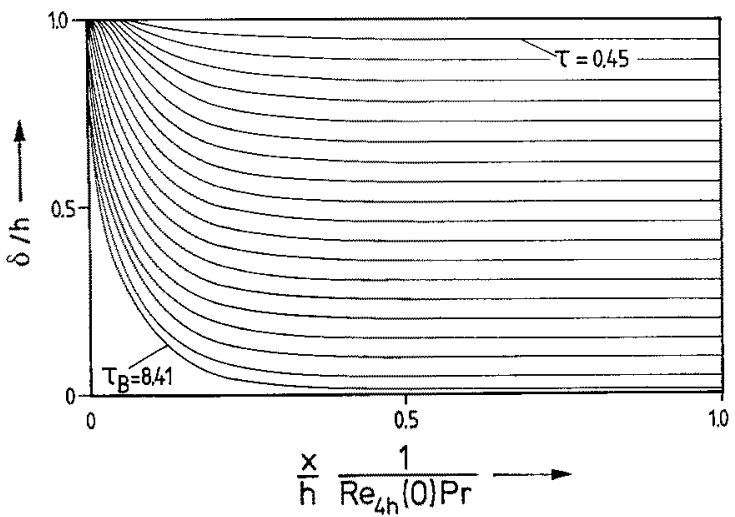

Fig. 11. Transient development of the frozen crust as a function of the axial coordinate for $B=10$ and $y=0.5$ (curves for equidistant time steps $\Delta \tau=0.45$ )

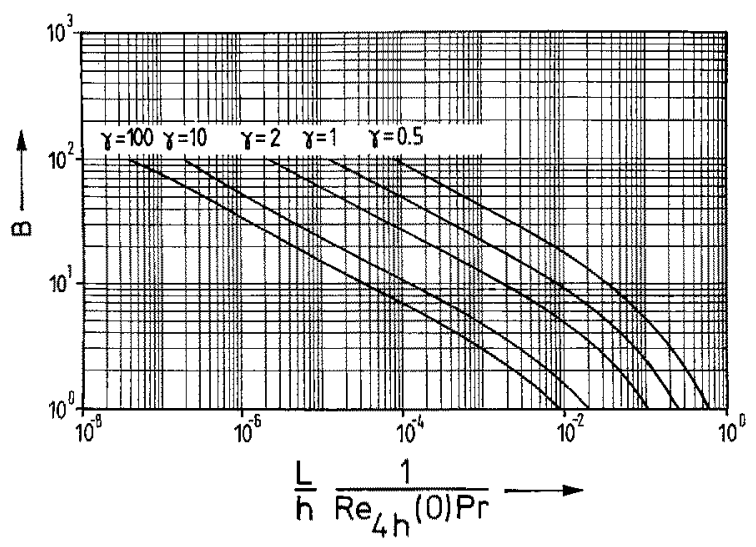

Fig. 12. Blockade diagram

(right hand side of the time line) from those regions, where steady-state solutions exist (left hand side of the line).

A similar diagram was developed by Sampson and Gibson [6] for the case of freezing in internal pipe flow with a constant wall temperature $(\gamma \rightarrow \infty)$. From Fig. 12 it is appar- ent that blockade in a convectively cooled channel having the same dimensionless channel length $\beta$ as a channel with constant wall temperature occurs for higher values of the cooling parameter $B$ (the curve for $\gamma=100$ shown in Fig. 12 coincides with the one for $\gamma \rightarrow \infty$ for constant wall temperature). This is due to the smaller amount of heat transfered by external convection from the channel walls to the ambient for a constant value of $B$ with decreasing values of $\gamma$.

\section{Concluding remarks}

By assuming a constant pressure drop across the channel, the freeze-shut of the planar duct, subjected to external convection, could be calculated approximately. According to the results of the present theoretical investigation, the following major conclusions may be drawn:

- The origin of the ice layers moves upstream during the growth of the frozen crust.

- A criterion was developed which predicts conditions under which blockade of the system will occur.

- The freeze-shut of a convectively cooled channel for low values of $\gamma$ is dominated by heat conduction in the frozen layer.

Finally, it should be noted that experiments dealing with the freeze-shut of a channel are not known in literature. It would be interesting to compare the given theoretical results with experimental data. Therefore, we hope that this study motivates the performance of an experimental investigation concerning the freeze-shut of a convectively cooled channel.

In the case of the freeze-shut of a circular pipe with constant wall temperature the agreement between theory and experiment was found to be satisfactory, as it was noted by Sampson and Gibson [6].

\section{Acknowledgement}

The support of this work by the Deutsche Forschungsgemeinschaft is greatly acknowledged.

\section{References}

1. Zerkle, R. D.; Sunderland, J. E.: The effect of liquid solidification in a tube upon laminar-flow heat transfer and pressure drop. J. Heat Transfer 90 (1968) 183-190

2. Lee, D. G.; Zerkle, R. D.: The effect of liquid solidification in a parallel plate channel upon laminar-flow heat transfer and pressure drop. J. Heat Transfer 91 (1969) 583-585

3. Kikuchi, Y.; Shigemasa, Y.; Oe, A.; Ogata, T.: Steady state freezing of liquids in laminar flow between two parallel plates. J. Nucl. Sci. Technol. 23 (1986) 43-55

4. Weigand, B.; Beer, H.: Liquid solidification in a parallel plate channel upon laminar-flow heat transfer: The stationary case. Wärme- und Stoffübertrag. 26 (1991) 233-240

5. Weigand, B.; Beer, H.: Transient freezing of liquids in forced laminar flow inside a parallel plate channel. Wärme- und Stoffübertrag. 27 (1992) 77-84 
6. Sampson, P.; Gibson, R. D.: A mathematical model of nozzle blockade by freezing. Int. J. Heat Mass Transfer 24 (1981) $231-241$

7. Des Ruisseaux, N.; Zerkle, R. D.: Freezing of hydraulic systems. Can. J. Chem. Eng. 47 (1969) 233-237

8. Myint-U., T.; Debnath, L.: Partial differential equations for scientists and engineers. New York, Amsterdam, London: North Holland 1987

9. Hsu, C. J: Laminar flow heat transfer in circular or parallelplate channels with internal heat generation and the boundary condition of the third kind. J. Chin. Inst. Chem. Eng. 2 (1971) $85-100$
10. Shah, R. K.; London, A. L.: Laminar flow forced convection in ducts. Adv. Heat Transfer. New York, San Francisco, London: Academic Press 1978

Dr.-Ing. B. Weigand

Dipl.-Ing. G. RuB

Fachgebiet Techn. Thermodynamik

TH Darmstadt

Petersenstraße 30

W-6100 Darmstadt, FRG

Received March 4, 1992 